\title{
Modelling Norm Scores with the cNORM Package in $\mathbf{R}$
}

\author{
Sebastian Gary ${ }^{1}$, Wolfgang Lenhard ${ }^{1, *}$ (i) and Alexandra Lenhard ${ }^{2}$ (i) \\ 1 Department of Psychology, University of Wuerzburg, 97070 Würzburg, Germany; Sebastian.Gary@gmx.de \\ 2 Psychometrica, 97337 Dettelbach, Germany; lenhard@psychometrica.de \\ * Correspondence: wolfgang.lenhard@uni-wuerzburg.de
}

check for

updates

Citation: Gary, S.; Lenhard, W.; Lenhard, A. Modelling Norm Scores with the cNORM Package in R. Psych 2021, 3, 501-521. https://doi.org/ $10.3390 /$ psych 3030033

Academic Editor:

Alexander Robitzsch

Received: 25 July 2021

Accepted: 26 August 2021

Published: 30 August 2021

Publisher's Note: MDPI stays neutral with regard to jurisdictional claims in published maps and institutional affiliations.

Copyright: (c) 2021 by the authors. Licensee MDPI, Basel, Switzerland. This article is an open access article distributed under the terms and conditions of the Creative Commons Attribution (CC BY) license (https:// creativecommons.org/licenses/by/ $4.0 /)$.

\begin{abstract}
In this article, we explain and demonstrate how to model norm scores with the cNORM package in $\mathrm{R}$. This package is designed specifically to determine norm scores when the latent ability to be measured covaries with age or other explanatory variables such as grade level. The mathematical method used in this package draws on polynomial regression to model a threedimensional hyperplane that smoothly and continuously captures the relation between raw scores, norm scores and the explanatory variable. By doing so, it overcomes the typical problems of classical norming methods, such as overly large age intervals, missing norm scores, large amounts of sampling error in the subsamples or huge requirements with regard to the sample size. After a brief introduction to the mathematics of the model, we describe the individual methods of the package. We close the article with a practical example using data from a real reading comprehension test.
\end{abstract}

Keywords: regression-based norming; continuous norming; inferential norming; data smoothing; curve fitting; percentile estimation

\section{Introduction}

Psychological tests are widely used instruments for measuring a variety of constructs such as intelligence, reading ability or personality. In most cases, a test raw score per se is not sufficient to assess the ability or personality trait of the test taker. Instead, the achieved raw scores have to be compared and interpreted in relation to an adequate reference population [1]. Since critical life decisions such as school placement or diagnosis of mental retardation are based on such tests, psychological tests need to supply high quality norm scores [1,2]. Unfortunately, the exact distribution of test raw scores in the target population is usually unknown; therefore, the norm scores cannot be determined directly. Instead, they must be derived from test results of a norm sample, that is, a representative subsample of the target population, using statistical tools.

Over time, different norming approaches have been developed and used. For example, modelling the course of distribution parameters such as mean and standard deviation over age was used already early on in intelligence test construction (see [1,3-6] for an overview). Subsequently, using the regression formula for the mean and the standard deviation, it was possible to estimate the norm score at any age. This approach however is based on very strong assumptions, e.g., normality, and it produces huge deviations especially at the upper and lower bounds of a scale. Moreover, mean and standard deviation were estimated independently, thus losing statistical power. This modelling approach was extended by including joint link functions and further parameters such as skew or by modelling nonnormal distributions [2]. Other approaches focus on modelling single percentile curves over age via quantile regression [7]. Recently, regression-based approaches to directly model the raw score distributions $[3,8]$ have become more and more popular. One of them is cNORM [9], which was implemented in the R package of the same name. In the following section, we present the cNORM approach and its mathematical background. Moreover, the necessary steps for computing norms with the cNORM R package are explained and, finally, the norming process is demonstrated on the example of a concrete data set. We do, 
however, start this article with a short general introduction about norm scores and their relevance for decisions based on psychological tests.

\section{Relevance of Norm Scores in Psychological Testing}

To characterise the current state of methodological development in the field of norming, we would like to draw an analogy to the development of classical test theory (CTT) and item response theory (IRT). While, at present, there is a convergence between the development of both approaches and they have been perceived as rather complementary [10] for a long time, IRT used to be seen as the more elaborate (but less used) framework for test development. To our understanding, a major reason for the perceived superiority of IRT was the simplistic assumption in former CTT that-apart from the measurement error-the distribution of the raw scores directly reflects the distribution of the ability or trait to be measured. Consequently, the major concern was to determine the error variance entailed in the raw score in order to infer the "true score" of a person or at least its confidence interval. In this sense, the "true score" approach of CTT leads to purely data-driven processing, without further explanations on how the test raw score results from the latent ability of a person. IRT in contrast always relied on the assumption that there is a probabilistic relationship between the latent ability of a person and the characteristics of an item. The test score does not directly represent the latent trait but the probabilistic outcome of the interaction between a person's ability and the item's difficulty-at least in the case of a 1PL IRT model.

In our view, calculating norm scores is far from being well enough understood and investigated so far. This shortcoming might be a consequence of the fact that many test constructors view norm scores as only referring to the manifest raw score distribution of a test scale. From this perspective, a norm score merely represents the location of a measured raw score in an empirical cumulative distribution function (eCDF); that is, it only conveys the information about the percentile rank regarding the eCDF of the raw scores in the norm sample. The mathematical processing of the eCDF therefore is usually limited to simple $z$-standardisation of the raw scores (plus linear scaling where required) in case of normality or to an inverse normal rank-based transformation (INT) of the raw scores to approximate normality (e.g., [11]). To put it yet another way, the significance of norm scores is limited to a manifest frequency information. Contrasting this notion, most test users probably see norm scores-at least implicitly—as an indicator of a latent trait, as for example in the case of the IQ scores in intelligence testing.

The notion that norm scores also represent latent traits is by no means an oversimplified assumption of psychometrically unskilled test users but is actually grounded in the definition of measurement itself. Measurement is defined as the homomorphous assignment of a numerical relative to an empirical relative (e.g., [12], p. 47), or, with regard to psychometrics, numbers have to be assigned to traits in a way that the original relationship between the traits of different persons is preserved in the numbers. In the case of psychometric tests, the latent traits of different persons lead to different test scores. The numerical relations between the different test scores should therefore reflect the relations between the traits of the persons. Test raw scores do not necessarily reflect all relations between latent traits. For example, in many instances, they do not reflect the true intervals between latent traits. Moreover, they cannot be interpreted by themselves due to the missing frame of reference. However, what is represented in the eCDF of raw scores in the norm sample is the rank information, that is, information about the raw score that is expected for a certain rank of the latent trait [13]. It should be noted here that just as in IRT, this is a relationship between the latent ability of a person and an expected outcome; however, unlike IRT, it is not the outcome of a single item but of the whole scale. If the rank information derived from the eCDF is projected onto a normal distribution (which is exactly what is achieved via INT) and the latent trait is in fact normally distributed, then the derived norm scores do represent the true intervals between latent traits. Therefore, norm scores derived via INT from the eCDF of the raw scores in the norm sample do not only represent frequency 
information of the raw score distribution. Instead, the relations between two different norm scores-apart from the individual measurement error-necessarily reflect the relations between the underlying latent traits as well.

Unfortunately, merely collecting data within a large norm sample and using INT to determine norm scores nevertheless entails numerous sources of error [13]. First, sampling error contained in the raw scores is fully preserved in the norm scores. Therefore, to avoid imbalances in the data, each norm sample has to be representative and of a sufficient size. Second, in cases of tests that span a large age range, subsamples for different age groups must be collected, in order to control for the development of the latent ability across age. Since norm scores are usually determined for each of these groups separately, very large sample sizes are needed, making test construction utterly expensive. However, imbalances between subsamples can nonetheless occur, and the trajectories of the percentiles across age can exhibit jagged or even implausible courses (e.g., older children obtaining better norm scores for the same raw score than younger children, when normally a continuous increase in performance would be expected over age). Third, in many cases (e.g., in educational testing), it is hardly even possible to cover the complete age range with subsamples of sufficiently narrow intervals. Therefore, in many cases either the norm scores are extremely imprecise (e.g., the same scale used for a whole cohort or grade) or there are large gaps between the subsamples (e.g., tests normed only at the end of each school year). The more a person's age deviates from the mean age of the respective subsample, the larger the bias becomes in the norm score [8]. Finally, norm scores can only be specified for raw scores, which indeed occurred in the norm sample. In summary, conventional norming, that is, INT of the eCDF per subsample, leads to a huge amount of additional errorbesides individual measurement error-contained in the norm scores $[1,8,13]$. Therefore, the central assumptions of measurement are nevertheless frequently violated if norm scores are derived in an overly simplistic way.

We would therefore like to present a norming procedure (Implementation: $\mathrm{R}$ package cNORM; [9]), which is able address the abovementioned drawbacks, thereby substantially reducing the error variance usually introduced by the norming procedure while simultaneously requiring much smaller samples [13]. We suggest to generally model the relation between a latent trait and the expected test raw score in the norming process with smooth functions, both per age and across age. In the simplest case, with a single norm sample, simple polynomial regression is used to model the monotonic function between raw scores and norm scores. In the case of additional explanatory variables such as, for example, age, a hyperplane is adjusted to the three-dimensional data map of person location (i.e., the rank information), raw score and explanatory variable $[1,6,8]$. The hyperplane is adjusted by drawing on polynomial regression as well and represents a continuous statistical model extracting the functional relation between latent trait, raw score and explanatory variable from the manifest data. By drawing on the complete data set, imbalances of distinct subsamples are smoothed, thus reducing local violations of representativeness and raising statistical power. For a detailed description of the types of norming errors, please see [13], where we could also show that continuously modelled norm scores more closely reflect the latent trait as compared to conventional test norms, retrieved via INT.

The approach makes only sparse assumptions on the nature of the latent trait and the data: First, we assume the existence of a latent trait, which is normally distributed at each level of the explanatory variable. Second, this latent trait interacts with the items of a scale, which-depending on the features of these items-leads to an expected raw score of each single test person and to an expected distribution of raw scores in the population. Note that this raw score distribution is not at all restricted to normality. Extreme floor and ceiling effects might, however, at some point be hard to model. Third, norm scores derived via INT from the eCDF mirror the normally distributed latent trait. Fourth, we further assume a bijective relationship between the norm scores and the expected raw scores at each level of the explanatory variable; that is, the relationship must be monotonic (e.g., higher latent traits consistently go along with higher expected raw scores or lower 
expected error rates at a fixed level of the explanatory variable). Finally, in the case of covariance between a latent trait and explanatory variable, the percentile curves must develop continuously and systematically, but not necessarily monotonically, across the explanatory variable. For example, the average raw scores of a test scale measuring fluid reasoning increase from childhood to early adulthood, but later, they decrease. Importantly, no further presumptions on the nature and course of this development are made. We outline the mathematical background of the procedure in the following section.

\section{Theoretical Background: The Rationale of cNORM}

As already described above, the cNORM R package aims at generating continuous test norms for psychometrics and biometrics via modelling the higher order three-dimensional relationship between the location $\theta$ (e.g., the latent trait, expressed as age-specific norm score), the explanatory variable $a$ (e.g., age or grade) and the expected test raw score $r$ through Taylor polynomials [1,8] (Figure 1). Moreover, it delivers methods for analysing the model fit of the used regression-based model. While the procedure was developed for generating continuous norms depending on explanatory variables such as age or grade in performance assessment, it can also be applied to physiological measurements such as body weight or height with both continuous and discrete explanatory variables (e.g., age, sex or test mode).
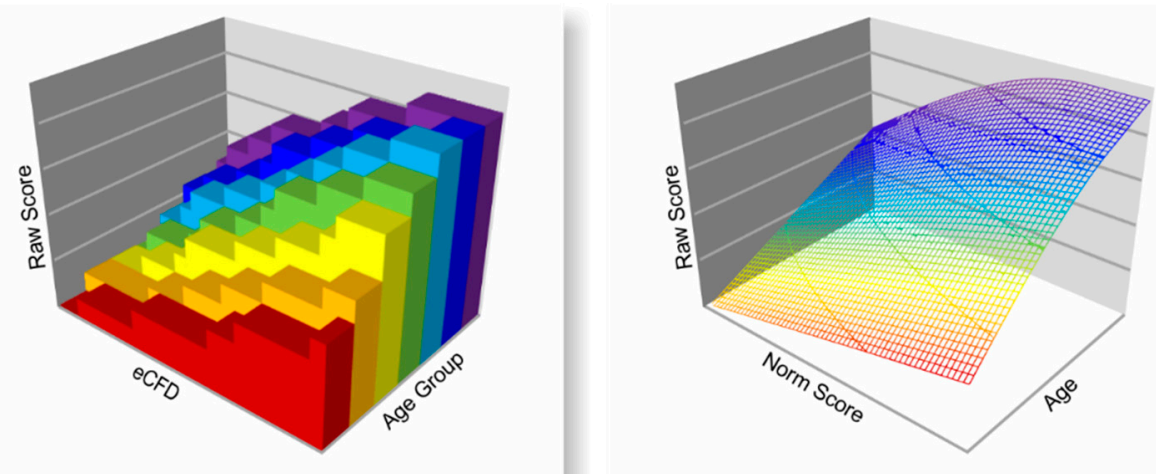

Figure 1. Visualisation of the norming approach as three-dimensional hyperplane fitting.

\subsection{Norm Scores and Taylor Series}

The main idea behind the norming approach used in CNORM is to consider the expected raw score $E(r)$ as continuous and a sufficiently often differentiable function depending on the person's latent parameter $\theta$ (e.g., his or her reading ability) and the explanatory variable $a$ (e.g., age) $[1,8]$. Therefore, we can formally specify the function:

$$
E(r)=f(\theta, a) .
$$

This kind of modelling approach covers the idea that a person's expected test score is caused by the interaction between a person's latent trait or ability and a certain test scale but additionally depends on her or his value of the explanatory variable. Since the functional relationship is assumed to be continuous and sufficiently differentiable, according to Taylor's theory, the function values around a given point $P\left(\theta_{0} ; a_{0}\right)$ can be expressed as such (more strictly, the infinite Taylor series converges only for values $\theta$ and $a$ within a certain radius around the point $P\left(\theta_{0} ; a_{0}\right)$. In practice, however, it has been shown that for many applications (e.g., data of psychological or physiological tests) the functional relationship can be approximated extremely well over a sufficient range)

$$
E(r \mid \theta, a)=\sum_{i, j=0}^{\infty} \frac{1}{i ! j !} \frac{\partial^{i+j} f\left(\theta_{0} ; a_{0}\right)}{\partial \theta^{i} \partial a^{j}}\left(\theta-\theta_{0}\right)^{i} \times\left(a-a_{0}\right)^{j}
$$


where $\partial^{i} \theta$ and $\partial^{j} a$ are the $i$-th partial derivation with respect to $\theta$, respectively, and the $j$-th derivation with respect to $a$. In other words, the function $E(r \mid \theta, a)$ can be expressed as the infinite sum of polynomials in $\theta$ and $a$. Since the infinite Taylor series converges to a finite value, namely the value of the raw score function, the individual summands and, therefore, the polynomial coefficients must become small very quickly, while the powers $i$ and $j$ increase. Hence, the original function should be approximated sufficiently well, if the expansion of the Taylor series is stopped after a certain number of steps $k$. In doing so, the functional relationship between the expected raw score, latent variable and explanatory variable can be approximated by a finite polynomial expression. Since the coordinates of the fixed $P\left(\theta_{0} ; a_{0}\right)$ as well as the derivatives at this point are constants, the previous equation can be simplified to

$$
E(r \mid \theta, a)=\sum_{i, j=0}^{k} c_{i, j} \theta^{i} a^{j}
$$

with the constants $c_{i, j}$ denoting the polynomial coefficients of the powers and interactions of $\theta$ and $a$. Therefore, the function can be approximated as a finite polynomial in two variables. To approximate the coefficients and determine the polynomial as precisely as possible, multiple regression is used on a representative norming sample for a given test (Figure 1 and following section) in order to select those powers of $a, \theta$ and their interactions up to the power parameter $\mathrm{k}$ that capture most of the variance in the norming sample.

Subsequently, the resulting regression model can be used to estimate a person's norm score $\hat{\theta}$ given his or her actual test score $r$ and the value of the explanatory variable $a$ by solving the equation

$$
r=\sum_{i, j=0}^{k} c_{i, j} \hat{\theta}^{i} a^{j}
$$

for $\hat{\theta}$. By doing so for a specific range of raw scores and values of the explanatory variable, norm tables can be generated and added to the test manual. Interpreted from a graphical point of view, the approach corresponds to fitting a hyperplane to the three-dimensional data map consisting of raw scores, values of the explanatory variable and person locations for every person in the norm sample. Figure 1 illustrates this graphical interpretation by comparing discrete mapping between person location and raw score for each individual age group (left side) with the corresponding three-dimensional functional relationship:

In summary, the cNORM norming approach reduces the problem of test norming to a model selection problem: To find adequate test norms, it is necessary to determine the coefficients of Equation (4) and, therefore, to find a polynomial regression model describing the norming sample as precisely as possible with the minimal number of predictors.

\subsection{Finding an Adequate Model}

Since multiple regression is used to obtain an approximation of the finite Taylor series, it is not clear, which specific powers and interactions of person location and explanatory variable do have a relevant influence on the raw score and, therefore, should be included as predictors of the statistical model. Moreover, adding an increasing number of predictors to the regression model, the procedure can lead to overfitting and to norming errors consequently. To avoid these and other problems of model selection, cNORM uses the best subset regression approach [14] based on a branch-and-bound-algorithm by Miller [15] as implemented in the R package leaps [16-18]. Given a data set together with all possible predictors, that is, all possible powers and interactions up to the maximum exponent $k$, the subset regression approach returns a regression model, which describes the given norm sample as well as possible with a minimal number of predictors. The predictors correspond to the coefficients of powers and interactions of person location and explanatory variable as described in Equation (4) for a fixed parameter $k$. Since practical applications have shown that higher values do not lead to better model fit in most cases [8,13], cNORM uses $k=4$ by default, and therefore, no higher powers and interactions than four are included 
in the regression equation. Moreover, the risk of overfitting increases with the number of included predictors. Therefore, we recommend using $k=4$ as a default for starting the norming process and including higher powers and interactions only in cases where adequate model fit cannot be reached with this parameter. cNORM also contains tools to assess the possible effects of over- and under-fitting via methods of cross validation. In the case of overfitting, $k$ should be reduced to 3 or even 2 , or the age power parameter should be reduced independently from the location power parameter.

\subsection{Norm Score Approximation}

After finding an adequate regression model, it is necessary to compute a solution of Equation (4) with respect to $\hat{\theta}$. From a mathematical point of view, solving Equation (4) for $\hat{\theta}$ is equivalent to finding the roots of a polynomial in one variable:

$$
r-\sum_{i, j=0}^{k} c_{i, j} \hat{\theta}^{i} a^{j}=0
$$

Instead of trying to solve (5) analytically, cNORM uses a numerical approach, more precisely, methods of numerical optimisation, by reformulating the problem of finding the roots into a minimisation problem. For this purpose, the left-hand side (5) is squared, and the resulting optimisation problem takes the following form:

$$
\min _{\hat{\theta}}\left(r-\sum_{i, j=0}^{k} c_{i, j} \hat{\theta}^{i} a^{j}\right)^{2} .
$$

Since squaring a real-valued function does not change the position of the function roots, every solution (5) is also a global solution of (6). If at least one real-valued solution of (5) exists, then the same also holds true for (6). Furthermore, every solution of (6) represents one of the desired solutions of Equation (5), since the minimised function (6) is non-negative valued. For (6), cNORM uses the optimize function of the R package stats [19]. Since the approach is based on a numerical procedure rather than an analytical solution, the resulting norm score does not reach the desired function value of zero exactly but with a sufficiently high precision. In case (5) does not have any root or the solution does not belong to the norm score range of the norm sample, cNORM returns the norm score within the range, which minimises Equation (6) and, therefore, approximates the desired norm score (for more detailed information about the exact implementation and optimisation algorithm used, see [9]).

\section{Generating Continuous Test Norms with cNORM}

To generate test norms using the cNORM approach with the corresponding software package, several steps are necessary. The three steps include:

1. Data preparation and modelling

2. Model validation

3. Generating norm tables

In the following section, these three steps are described and explained in detail both from a theoretical point of few as well as from the side of their practical application. Information about the installation of RStudio and the cNORM R package can be found here: [20]. We will first explain the steps of the process in more detail, before demonstrating a comprehensive function that applies all steps in one process.

\subsection{Data Preparation and Modelling}

The basis of every norming process is a sufficiently large and representative norming sample. Therefore, some authors recommend using random sampling but emphasise that a stratification of the norm sample could be necessary in the case of unbalanced data 
sets, especially when using small samples [13]. For example, to norm an intelligence test for a broad age range, it is necessary to stratify the norm sample with regard to the explanatory age variable, since an unbalanced set could lead to norming errors, especially in the underrepresented age intervals. One major advantage of using statistical continuous norming models instead of simply applying inverse normal transformation per age group is the increased statistical power, since the modelling draws on the complete data set. Consequently, smaller samples are already sufficient for obtaining a higher norm score precision compared to traditional norming approaches. For instance, with cNORM, 100 cases per age group already lead to more precise norms as compared to 250 cases in traditional norming [13]. Choosing a modelling approach like cNORM can thus not only close the gaps between norm tables but can also render test norming projects more cost efficient while improving data quality. For more detailed information to the process of representative sampling, see [21,22].

After establishing a representative norm sample, the data must first be imported in the $R$ environment. It is advisable to start with a simply structured data object of type data frame or even a numeric vector containing raw score and explanatory variable. Missing data in the norm sample should be excluded before the actual norming process. Since the explanatory variable in psychometric performance tests is usually age, the term 'age' as well as the shortcut $a$ is used in the following to refer to the explanatory variable. In fact, the explanatory variable is not necessarily age but must in any case be an interval or nominal variable. Finally, a grouping variable is necessary to break down the norm sample into smaller subsamples (for example grades or age groups). The method is relatively robust to changes in the granularity of the group subdivision and has little effect on the quality of the norm scores [8]. As a rule of thumb, the more the explanatory variable covaries with the measured test score, the more groups should be formed [13]. Consequently, age groups need not necessarily be equidistant, or each contain a constant number of cases, but rather should increase in granularity at those areas where fast development of the latent ability occurs. By default, cNORM assigns the variable name 'group' to the grouping variable. To divide the norming sample into adequate groups, the 'getGroups' method can be used. By default, the function divides the sample into groups of equal size and returns a vector containing the group mean of the explanatory variable for every subject.

The next step is to rank each person in each group by using the 'rankByGroup' function, which returns percentiles and performs a normal-rank transformation using $T$-scores $(M=50, S D=10)$ by default $(z$ - or $I Q$-scores can also be used, and it is possible to define arbitrary scales by specifying mean and standard deviation). By doing so, every individual subject in the test sample is ranked in comparison to all other subjects in the same group. Different ranking methods (RankIt; Blom; Van der Warden; Tukey; Levenbach; Filliben; Yu and Hang) can also be used; however, by default RankIt was chosen. Instead of using a grouping variable respectively dividing the age variable into distinct age groups, one can also use the 'rankBySlidingWindow' method. This method draws on the continuous age variable and uses a sliding age interval to rank each subject in the norm sample relative to all other subjects whose age does not differ by more than half the width of the age interval from the age of the ranked subject. Grouping into distinct subgroups with the 'getGroups' function is thus not necessary. The width of the sliding age interval is set by specifying the function parameter ' $w i d t h$ '. For example, setting 'width $=0.5$ ' by using age as a continuous variable in the unit 'year' means that the width of the age interval is six months, and therefore, every subject is assigned a rank based on all other subjects no more than three months younger or older than the person itself. The ranking by sliding window can be seen as a special case of ranking by group but with every subject being ranked with respect to all other subjects within a symmetric interval around the subject's age value. Both ranking functions add the two columns 'percentile' and 'normValue' to the data, which are necessary for further computations. Moreover, descriptive information for every group such as group mean and standard deviation is added to the data. 
The next step initiates the actual modelling process by determining a polynomial to express the raw score as a function of the estimated latent person parameter $\hat{\theta}$ and the explanatory variable a. As described in Section 2 of this manuscript, we believe that the norm score assigned to a certain raw score reflects the estimated latent person parameter $\hat{\theta}$. In the following section, we do, however, refer to the norm scores as $l$ for location instead of $\hat{\theta}$, because $l$ is the abbreviation used in the R package. To retrieve the model, all powers of the variables $l$ and $a$ up to a certain exponent $k$ together with all possible interactions are computed. To compute the desired powers, one should use the 'computePowers' method. Please note that using a power parameter $k$ means to compute $2 \times k+k^{2}$ new variables corresponding to the powers of $l$ and $a$ as well as their interactions. Therefore, the number of used predictors for modelling the regression function grows in a quadratic manner with respect to $k$. As a rule of thumb, $k$ should be chosen to be smaller than 5; hence, higher values can lead to the overadjustment of the model. Alternatively, the power parameter for location and age can be set independently, with higher values for location and smaller powers for age, since age trajectories often can be modelled by quadratic or cubic polynomials. By default, cNORM uses $k=4$ for both location and age, since practical application so far has shown a high goodness of fit with even a small number of predictors $[1,8,13]$, and it also provides methods for cross validation, verification of the model assumptions, visual model inspection and visualisation of percentiles to determine the optimal setting. Applying the method to the norm data adds $2 \times k+k^{2}$ columns to the data set representing the values of all possible predictors of the raw scores.

The complete process of data preparation, containing ranking and computing powers can also be performed by using the function 'prepareData'. Please note that when using this function, the names of the used grouping variable and raw score variable must be assigned to the parameters 'group' and 'raw', respectively.

To start the computation of the targeted regression model, the next step is to use the 'bestModel' function. There are several ways to use this function: By specifying $R_{\text {adjusted }}^{2}$ the function returns the model that satisfies this requirement with the smallest possible number of predictors. Instead, one can specify a fixed number of predictors, and the method in turn delivers the model with the highest $R_{\text {adjusted }}^{2}$ to this number of predictors. Finally, a specific regression formula can be specified, and cNORM fits the regression weights accordingly. Since in most cases it is not possible to determine in advance how well the data can be modelled, the default settings are four for the number of predictors and 0.99 for $R_{\text {adjusted }}^{2}$. If the targeted precision of the regression model in terms of $R_{\text {adjusted }}^{2}$ is not reached, the method returns the model with all $k^{2}+2 k$ allowed predictors. However, the threshold of $R_{\text {adjusted }}^{2}=0.99$ is no ultimate criterion for model selection, since it depends on the covariance between the explanatory variable and the latent ability as well as on the size of the norm sample. It might even be suitable to reduce this value to 0.95 to smooth the percentiles and to avoid model overfitting. This can usually be achieved by reducing the power parameter $k$ and/or the number of terms in the regression model.

\subsection{Model Validation}

The cNORM package contains a variety of methods for validating the resulting regression model. For this purpose, it is helpful to remember the main idea of the norming approach. From a graphical point of view, the resulting regression function represents a so-called hyperplane in three-dimensional space spanned by the three dimensions raw score, norm score and explanatory variable.

On the one hand, it is necessary to evaluate the model in terms of whether it is suitable to provide adequate test norms. If the resulting $R_{\text {adjusted }}^{2}$ is sufficiently high, the hyperplane usually models the manifest norm data over a wide range of all variables very well. However, since the approach is based on a Taylor polynomial, which usually has a finite radius of convergence, it is possible that there are age or performance ranges for which the regression function no longer provides plausible values. With a high $R_{\text {adjusted }}^{2}$ this boundary of convergence is reached at the outer edges of the age or performance range 
of the norm sample or even beyond. These limits are not due to the used method only but also because the underlying test scales have only a limited validity range within which they are able to map a latent ability to a meaningful numerical test score (e.g., because of floor and ceiling effects and in general due to skewness of the raw score distributions). Therefore, norm tables and norm scores should generally only be issued within the validity range of the model. In addition, even if extrapolation to extreme raw scores that were not reached once in the norm sample yields plausible norm scores, such extrapolation should be carefully considered. For determining the limits of the regression model and for identifying model violations, cNORM provides graphical and numerical methods to investigate the possible limitations of the computed model. First, the 'plotPercentile' function of cNORM can be used to visually check the model fit. This function plots the manifest as well as the approximated relation between raw scores and percentiles as functions of the explanatory variable. By default, the maximum and minimum raw scores reached in the norm sample are used as the upper and lower limits of the plotted raw scores, but the range of plotted raw scores can also be set manually by using the function parameters 'minRaw' and 'maxRaw'. It is important to make sure that the percentile lines do not intersect, because otherwise different percentile ranks would be assigned to the same raw score. As previously mentioned, intersecting percentile curves can occur when the regression model is extended to age or performance ranges that do not or only rarely occur in the norm sample. Moreover, intersecting percentile curves can be a sign of strong floor or ceiling effects in the test and are not necessarily due to the cNORM approach. From a mathematical point of view, intersecting percentile curves would implicate that the mapping from raw score to norm score is not unique for a fixed level of the explanatory variable. As a result, more than one norm score would be assigned to a given raw score, which would not only violate the model assumptions but also lead to problems in the practical application of norm scores. Second, one can use the 'plotRaw' function to compare fitted and manifest data for every group separately. The model fit is particularly good if all points are as close as possible to the bisecting line. However, it must be noted that deviations in the extremely upper and lower performance range often occur because, as already mentioned, the manifest data in these areas only rarely occur in the norm sample and are therefore afflicted with high measurement error. Alternatively, the 'plotNorm' method can be used, which delivers an equivalent plot as 'plotRaw' but uses norm scores instead of raw scores. Therefore, the plotted values should also approach the bisecting line as closely as possible. Both plots can be regarded as a graphical illustration of the accordance between predicted and observed values and, therefore, as a graphical visualisation of the model fit. Finally, to check the monotonicity of the mapping function between latent variable and raw score, the 'plotDerivative' method can be used, which plots the first-order derivative of the regression function with respect to $l$. This method should be used to check whether there is any age or performance range with a zero-crossing, indicating a violation of the monotonicity. Please note that for example zero-crossings in the upper age and performance range do not necessarily mean that the modelling has completely failed, but that the test scale loses its ability to differentiate in this particular measurement range. This is important when subsequently norm tables are calculated from the model, since these performance ranges should be excluded from the tables. Alternatively, cNORM provides a 'checkConsistency' method, which scans all age and performance ranges numerically to find any violation of the bijectivity in the regression function. We recommend using both methods to find any violation of the model assumptions, especially intersecting percentiles, but as well contra intuitive age progression and undulating percentile curves as a sign of overfit.

Furthermore, it is also necessary to evaluate the model in terms of how well it fits the norm sample and whether it shows any signs of over- or under-fitting. For validating the derived model from a more statistical point of view, the 'plotSubset' function delivers additional information about $R_{\text {adjusted }}^{2}$ and other information criteria, such as Mallow's $C_{P}$ or $B I C$, depending on the number of predictors with fixed parameter $k$. Different charts are provided if the 'type' parameter is changed (1: $R_{\text {adjusted }}^{2}$, $\left.C_{P}, 3: B I C\right)$. The method can 
be especially helpful in case the resulting model seems to be invalid because of violations of consistencies in one or more measurement ranges. In addition, these information criteria can be particularly helpful in detecting any signs of over- or under-fitting. Depending on the specific parameter, the method returns a visualisation of the chosen information criterion as a function of the number of used predictors. Moreover, cNORM provides the 'cnorm.cv' method, which is a validation function to obtain an impression of the quality of the norming process. To this end, the method splits the data set into a training set $(80 \%)$ and a validation set $(20 \%)$, computes norming models with increasing numbers of predictors starting with one predictor up to a specified number (parameter 'max') based on the training set, and compares the predicted with the observed scores of the validation set in terms of the root mean squared error (RMSE) of the raw score as well as the adjusted $R^{2}$ of the norm score. Moreover, the method returns a crossfit value which can be used to identify the possible effects of under- or over-fitting. Crossfit values lower than one indicate signs of underfitting, while values greater than one suggest overfitting. By setting the parameter 'repetition', the number of validation cycles, that is, the number of times the sample is split, and the cross validation is repeated can be specified. Please note that for every repetition it is necessary to rank the training and validation set as well as to compute regression models with increasing numbers of predictors. For example, repeating the validation cycle ten times with a maximal number of predictors of twelve, the norm sample must be divided and ranked ten times, and $10 \times 12=120$ regression models model must be computed Consequently, the computational effort increases rapidly with the maximum number of predictors and the number of repetitions, leading to high computational duration. As a rule of thumb, crossfit values within $[0.90 ; 1.10]$ are acceptable, while lower and, respectively, higher values should be seen as evidence of underfitting and, respectively, overfitting. Further research is necessary to investigate lower and upper bounds of crossfit values as indicators for under- and over-estimation in more detail

\subsection{Generating Norm Tables}

While the actual norming process provides a pure functional expression in a statistical sense, the cNORM package also contains methods to retrieve lists of norm scores for specific raw scores and, vice versa, raw scores for specific norm scores. Additionally, cNORM contains a variety of methods for the visualisation of norm curves.

First, cNORM provides the 'getNormCurve' method, which returns the fitted raw scores for a specific norm score. For example, specifying the norm score as $T=50$ in terms of a $T$-score, the method plots the raw scores assigned to a norm score of $T=50$ as a function of the explanatory variable (e.g., age). By default, the plotted range of the explanatory variable is limited by the corresponding range of the norm sample but can be specified using the parameters 'minAge' and 'maxAge' to set the desired values. Please note that the estimated norm scores can differ greatly from the true ones if the used age values are extrapolated to values that are not contained in the norm sample. Therefore, extrapolation should be used cautiously or at least be marked in the test manual.

Second, the 'plotNormCurves' method can be used to visualise norm curves for more than one norm score simultaneously. For example, setting the 'normList' parameter to ' $c(30,40,50,60,70)$ ' returns a visualisation of norm curves corresponding to the $T$-scores $30,40,50,60$ and 70 in one plot. In addition to the percentile curves mentioned above, the visualisation can also be used for checking the model validity, since intersecting norm curves would indicate a misspecification of the statistical model. Moreover, intersecting percentiles can emerge also due to floor and ceiling effects, for example, if the test cannot adequately discriminate in extreme performance ranges. In this case, the corresponding raw score should be assigned to a percentile range rather than to a single norm score to indicate that test scores in this performance range should be interpreted cautiously. This is especially important in extremely low norm ranges, since decisions about school placement and the like are often based on such norm scores. 
Third, the 'predictNorm' function can be used to predict the norm score for a single raw score given a specific age. For considering the limits of the model validity, the minimum and maximum norm score can be specified by setting the parameters 'minNorm' and 'maxNorm' to the corresponding values. Likewise, the 'predictRaw' method returns the predicted raw score for a specific norm score and age.

When it comes to test application and automatised scoring and interpretation of test results, the statistical model is completely sufficient to provide norm scores with a prespecified precision for any level of the explanatory variable. In real-world scenarios, however, manual scoring is still very common, which is why in most cases norm tables must also be provided for users. One of the major advantages of statistical norming models is the possibility to also decide on the granularity of the norm tables and to provide norm scores even for those raw scores or levels of the explanatory variable for which no manifest data are at hand [13]. In typical scenarios like achievement tests, the available norms are often referred to an entire grade level. By contrast, continuous norming methods like cNORM can, for example, provide norms per months or per week, theoretically even down to the exact day. Additionally, there are no missing values in the norm tables anymore, and there is even the possibility for cautious extrapolation to age or performance ranges not contained in the norm sample. To this end, cNORM provides functions to generate norm tables with either the assignment of norm scores to raw scores or vice versa: The 'normTable' method returns the corresponding raw scores for a specific age or vector of ages. Using the parameter 'step', the desired interval between two norm scores can be set. Furthermore, if a coefficient of reliability is entered, confidence intervals for the norm scores and the percentile ranks are also computed automatically.

In equivalence to the 'normTable' function, the 'rawTable' method can be used to assign predicted norm scores to a predefined series of raw scores at a certain age. The function is very useful in case the exact percentiles or the exact norm scores are to be determined for all raw scores that may occur in a given test scale and for a given range of the explanatory variable. By setting the parameter 'step', the desired precision for the raw scores can be specified. Since predicting the norm score for a given raw score and age requires an inversion of the regression function, with the latter being determined numerically, the computational of the 'rawTable' method increases with the higher precision and smaller step size.

\section{Step-by-Step Example: Continuous Norming of a Reading Comprehension Test}

To give a detailed example of the cNORM norming process following the alreadymentioned three necessary steps, the norming sample of the sentence completion subtest of a German reading comprehension test named ELFE 1-6 [23] is used in what follows. The data set is already included in the cNORM R package and can directly be retrieved. In the first step, the data is assigned to the 'data.elfe' variable, and the 'head' method is used to obtain a first impression of the data set (Figure 2).

\begin{tabular}{|r|r|r|r|}
\hline & personID & group & raw \\
\hline $\mathbf{1}$ & 197 & 2 & 0 \\
\hline $\mathbf{2}$ & 295 & 2 & 0 \\
\hline $\mathbf{3}$ & 1261 & 2 & 0 \\
\hline $\mathbf{4}$ & 1317 & 2 & 0 \\
\hline $\mathbf{5}$ & 1331 & 2 & 0 \\
\hline 6 & 239 & 2 & 1 \\
\hline
\end{tabular}

Figure 2. Structure of the included ELFE data set.

As can be seen, there is no age variable in the data set, only person ID, a raw score, and a grouping variable. In this case, the grouping variable also serves as a continuous explanatory variable since children were only examined at the very beginning and the 
exact middle of the school year during the test standardisation. For example, a value of 2.5 indicates that the corresponding participants were examined in the middle of the second grade. Another possibility would have been to examine children throughout the entire school year instead and to use the schooling duration as a continuous variable. To build the grouping variable, the first and second half of each year could, for example, be aggregated into one group, respectively. Since the participants in this sample are grouped already, it is not necessary to use the 'getGroups' method. In the data set, there are seven groups with 200 cases each, totalling 1400 cases:

\# Example dataset based on a reading comprehension test

data.elfe $<-$ elfe

\# Inspect the first six lines of the data set

head (elfe)

\subsection{Data Preparation and Modelling}

The next step is to apply the 'rankByGroup' method. Please note that the aforementioned 'rankBySlidingWindow' method is no valid alternative in this case, because the used explanatory variable is no continuous variable. The RankIt method is chosen as ranking method by setting the 'method' parameter to ' 4 '. In addition, $T$-scores with $M=50$ and $S D=10$ are chosen as norm scale. As already mentioned, other scales can be used by specifying the mean and standard deviation as a vector to the 'scale' parameter of the method (for example, IQ-scale can be used by setting 'scale $=\mathrm{c}(100,15)^{\prime}$ instead). If the entire sample is to be ranked without a grouping or explanatory variable, the optional parameter 'group' must be set to 'FALSE'. By doing so, the rank of every subject is determined with respect to the whole norming sample. If a grouping variable with a name other than "group" is to be used, the "group" parameter must be set to this very name:

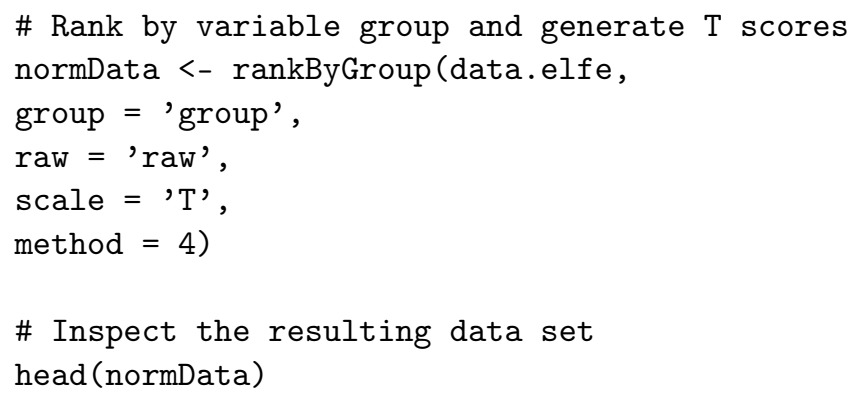

A short inspection of the resulting data set shows that various columns such as manifest percentiles, mean and standard deviation of the corresponding group were added to the data set, as previously described (Figure 3).

\begin{tabular}{|r|r|r|r|r|r|r|r|r|r|r|}
\hline $\mathbf{4}$ & personID & group & raw & percentile & $\mathbf{n}$ & $\mathbf{m}$ & md & sd & normValue \\
\hline $\mathbf{1}$ & 197 & 2 & 0 & 0.0125 & 200 & 7.32 & 7 & 4.351122 & 27.58597 \\
\hline $\mathbf{2}$ & 295 & 2 & 0 & 0.0125 & 200 & 7.32 & 7 & 4.351122 & 27.58597 \\
\hline $\mathbf{3}$ & 1261 & 2 & 0 & 0.0125 & 200 & 7.32 & 7 & 4.351122 & 27.58597 \\
\hline $\mathbf{4}$ & 1317 & 2 & 0 & 0.0125 & 200 & 7.32 & 7 & 4.351122 & 27.58597 \\
\hline $\mathbf{5}$ & 1331 & 2 & 0 & 0.0125 & 200 & 7.32 & 7 & 4.351122 & 27.58597 \\
\hline $\mathbf{6}$ & 239 & 2 & 1 & 0.0400 & 200 & 7.32 & 7 & 4.351122 & 32.49314 \\
\hline $\mathbf{7}$ & 379 & 2 & 1 & 0.0400 & 200 & 7.32 & 7 & 4.351122 & 32.49314 \\
\hline
\end{tabular}

Figure 3. Result of the inverse normal transformation of the manifest data, including manifest percentiles and norm scores ( $T$-scores) as well as group-specific descriptive data. The column 'normValue' will serve as the location 1 in the following. 
The last column contains the manifest norm scores with respect to the corresponding group, expressed as $T$-scores. These values and the grouping variable are used in the next step of the data preparation procedure to calculate powers and interactions. To this end, the method 'computePowers(normData)' is used. The function appends the resulting powers and interactions of norm score and grouping variable to the data set as columns (Figure 4).

\begin{tabular}{|c|c|c|c|c|c|c|c|c|c|c|c|c|c|c|c|}
\hline & A1 & L1A1 & L1A2 & L1A3 & L1A4 & A2 & A2 & L2A1 & L2A2 & [2A3 & L2A4 & $A^{3}$ & ${ }^{A 3}$ & L3A1 & L3A2 \\
\hline 27.585973 & 2 & 55.171945 & 110.34389 & 220.68778 & 441.37556 & 760.98589 & 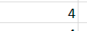 & 1521.9718 & 3043.9436 & 6087.8871 & $12,175.77$ & $20,992.54$ & 8 & $41,985.07$ & 0.14 \\
\hline 27.585973 & 2 & 55.171945 & 110.34389 & 220.68778 & 441.37556 & 760.98589 & 4 & 1521.9718 & 3043.9436 & 6087.8871 & $12,175.77$ & $20,992.54$ & & $41,985.07$ & $83,970.14$ \\
\hline 27.585973 & & 55.171945 & 110.34389 & 220.68778 & 441.37556 & 760.98589 & & 1521.9718 & 3043.9436 & 5087.8871 & 175.77 & 992.54 & 8 & $41,985.07$ & $83,970.14$ \\
\hline & 2 & 45 & 389 & 3778 & 556 & 889 & & .9718 & 9436 & .8871 & 5.77 & 2.54 & & 855.07 & 0.14 \\
\hline & 2 & 45 & & 3778 & & & 4 & & & & & & & 5.07 & \\
\hline 32.4 & 2 & 79 & 6 & 511 & 9.89023 & 5.8041 & 4 & 82 & 64 & 28 & .87 & 39 & & 2.78 & 56 \\
\hline 32.493139 & 2 & 64.986279 & 129.97256 & 259.94511 & 519.89023 & 1055.8041 & 4 & 2111.6082 & 4223.2164 & 8446.4328 & $16,892.87$ & $34,306.39$ & 8 & $68,612.78$ & $137,225.56$ \\
\hline
\end{tabular}

Figure 4. Prepared data set containing powers and interactions of location (manifest norm score) and age.

As mentioned, cNORM calculates powers and interactions up to an exponent of $k=4$ by default. Thus, a total of $4 \times 4+2 \times 4=16+8=24$ predictors are added to the data set as additional columns. To start the actual modelling process, the 'bestModel' function is used with a default stopping criterion of $R^{2}=0.99$. The resulting model contains four predictors plus an intercept and captures more than $99 \%$ of the variance of the norm data. For a more detailed summary, cNORM's 'summaryModel' can be applied to the norm model:

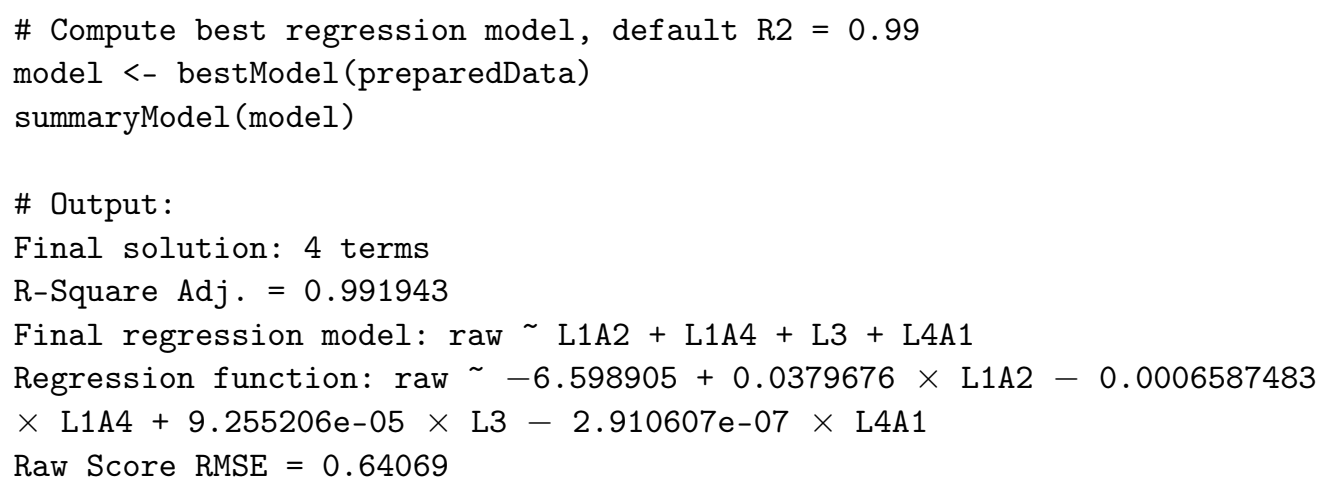

The final solution, as chosen by the best subset approach, contains four predictors, namely $l a^{2}, l a^{4}, l^{3}$ and $l^{4} a$, as well as an intercept. The resulting polynomial regression function in this case is as follows:

$$
r(l, a)=-6.599+0.0381 a^{2}-0.001 l a^{4}-2.912 l^{4} a
$$

Moreover, the deviation between observed and predicted raw scores seems to be sufficiently small with a root mean squared error of $R M S E=0.64069$. In summary, the resulting regression model seems to cover a great amount of the variance in the norm data combined with a small, global prediction error.

\subsection{Model Validation}

To obtain a first impression of the model fit in more detail, the 'bestModel' function automatically returns a visualisation of the observed and predicted percentile curves which can also be generated by using 'plotPercentiles(model)' (Figure 5).

The plot shows only a small deviation between observed and predicted values, which can be interpreted as a first sign for a good model fit. Jagged or curvy trajectories, which are most likely the result of sampling error, are smoothed, leading to a better estimation of the latent ability per age. Specifically, no intersecting percentiles curves are predicted. Therefore, the mapping from raw score and explanatory variable to norm score seems to be unique within the observed range of age and raw scores. The opposite case would be a 
sign that the computed model is not valid for describing the norming sample. By default, the range of the explanatory variable is set to the minimum and maximum values of the used data set. As already mentioned before, we recommend using the computed model only for predictions within the range of the norm sample, since extrapolation can lead to large prediction errors. Another way to inspect the model is to compare the observed and fitted data in terms of the raw score with plotRaw(model). The method plots the observed against the predicted raw score and delivers information about the model fit in terms of correlation and RMSE (Figure 6).

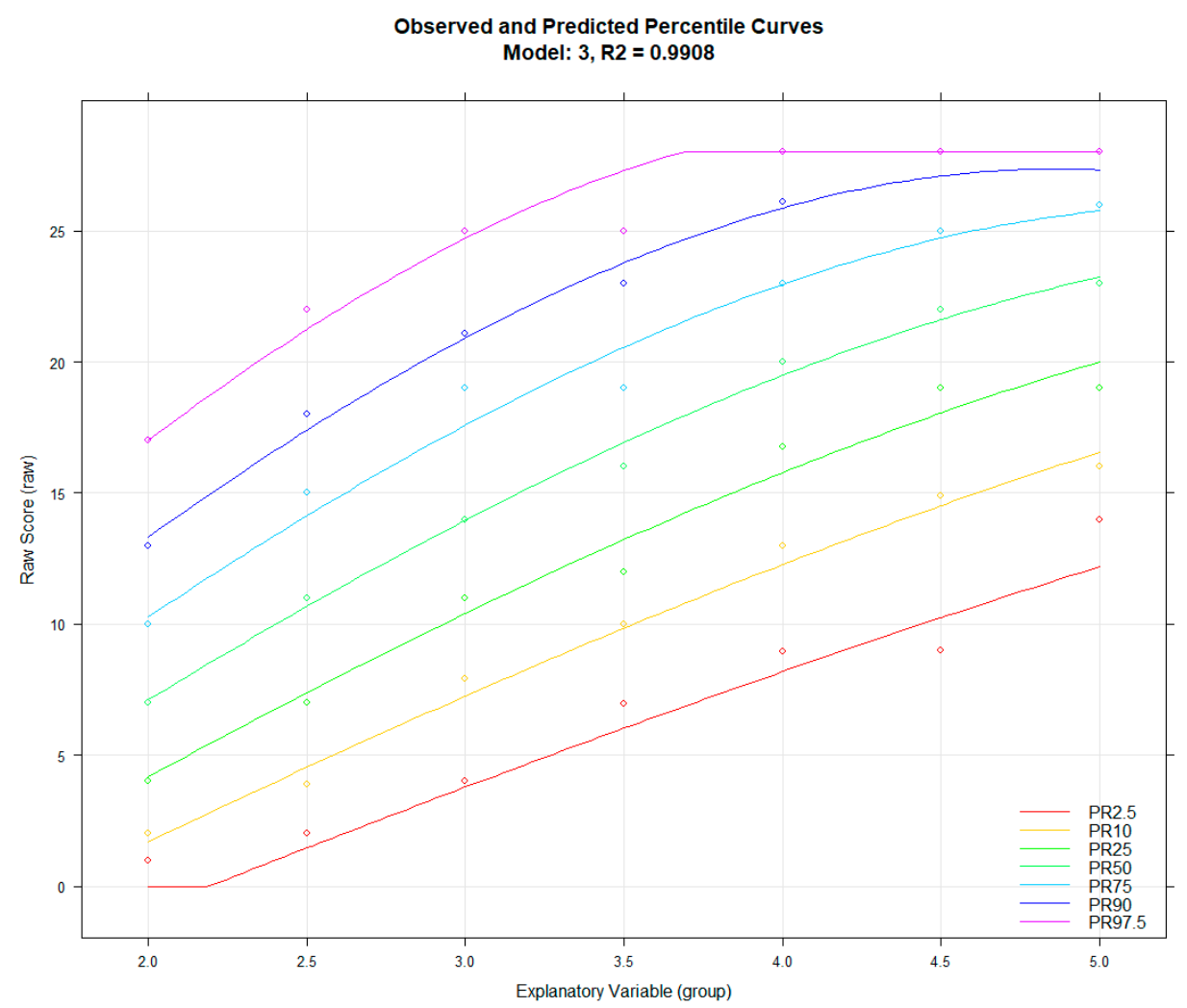

Figure 5. Observed (circles) and predicted percentile curves.

The visual inspection reveals only a small deviation between observed and predicted raw scores in line with a high correlation $(r=0.9954)$ and a small root mean squared error $(R M S E=0.6835)$. The 'plotNorm' functions deliver a nearly identical result. While the regression model seems to deliver nearly perfect predictions for the average performance range around $T$-scores of 50, higher deviation can be observed in the strongly belowaverage range of $T<30$. Although the model seems to fit quite well overall in this case, the example still shows that the goodness of fit usually varies within the targeted measurement range. Higher deviations in the more extreme performance ranges may also be due to the fact that many tests contain only a low number of items that are very easy or very difficult. For investigating the model consistency in terms of monotonicity, the function checkConsistency(model) method may be used. The function examines whether model assumptions are violated using the derivative of the regression function and returns information as well as hints for exploring them further. In the case of the model of the example, it issues the following warning: "Violation of monotonicity at age 5. At least 1 violations of monotonicity found within the specified range of age and norm score. Use 'plotNormCurves' to visually inspect the norm curve or 'plotDerivative' to identify regions violating the consistency. Rerun the modelling with adjusted parameters or restrict the valid value range accordingly. Be careful with horizontal and vertical extrapolation. The original data for the regression model spanned from age 2 to 5 , with a norm score range from 21.93 to 78.07. The raw scores range from 0 to 28." 

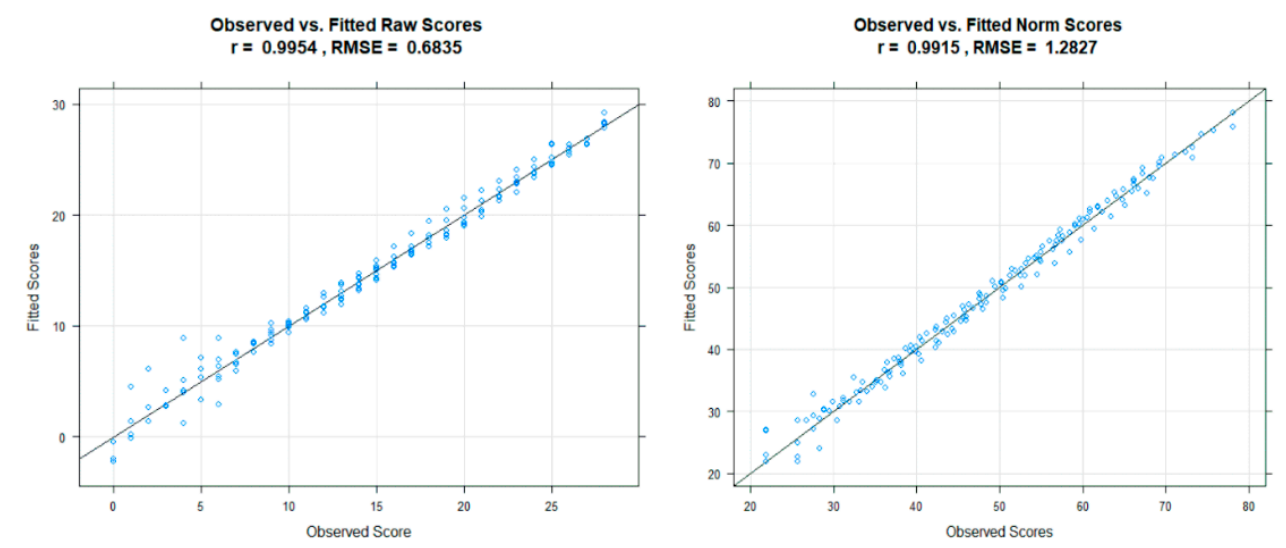

Figure 6. Observed and predicted raw and norm scores.

The procedure indicates a possible violation of the model assumptions in the fifth grade. (Please note that the variable $a$ normally denotes age, but that in this particular case the grade level was used instead.) For further investigation, the 'plotDerivative' function can be used, which returns a visualisation of the derivative of the regression model with respect to $l$. In order to ensure a bijective relation of norm and raw scores, the slope must be either consistently positive or negative at all age and performance ranges. Zero-crossings indicate intersecting percentile lines. Thus, the yellow areas in this map indicate model violations or regions, where the model is less precise. In the current example, this is the case at high performance levels in the fifth grade, resulting from a ceiling effect of the test scale (Figure 7 , indicated in red in the right upper edge).

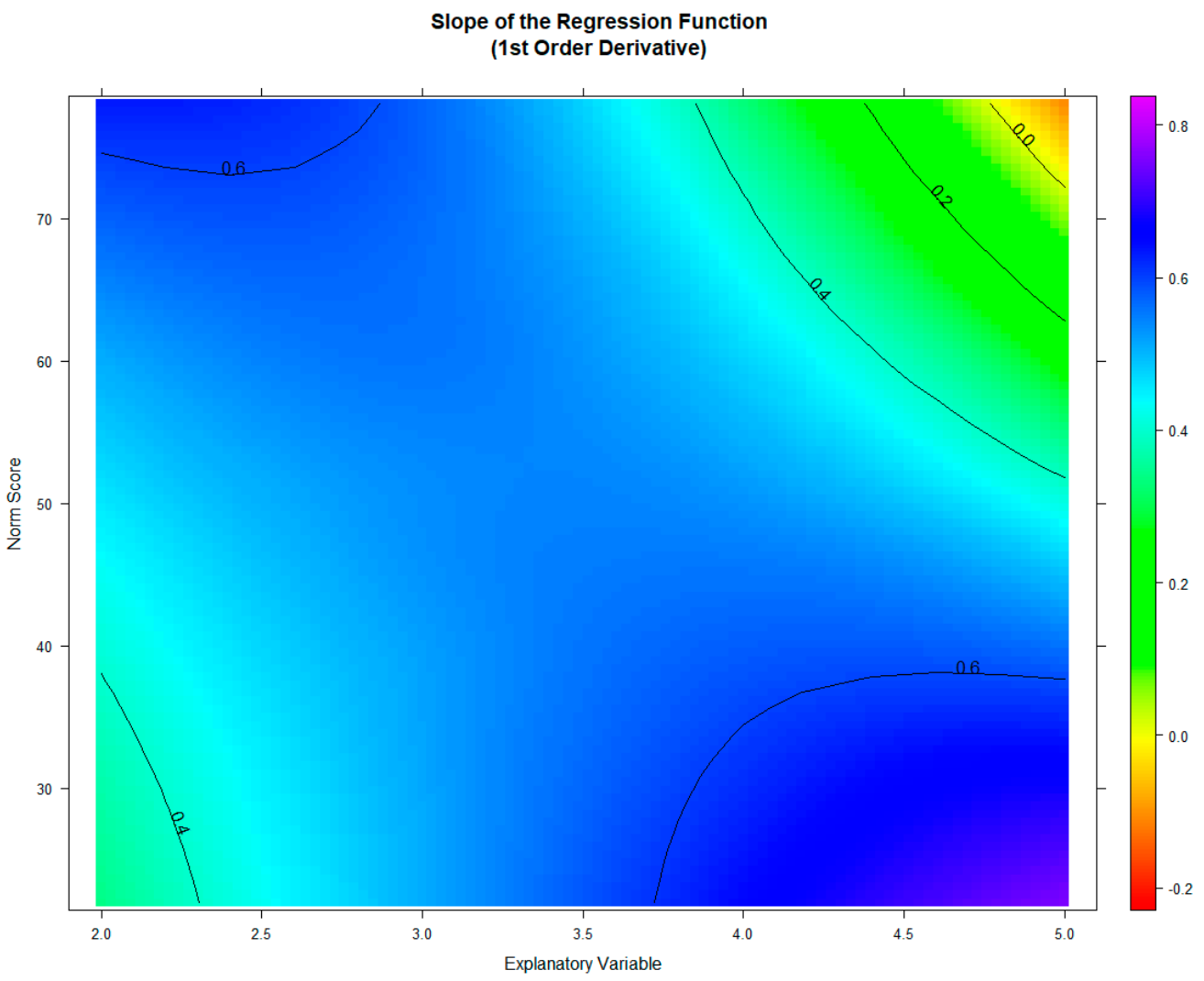

Figure 7. Derivative of the regression function.

Consequently, in the fifth grade and in the highest performance range, higher raw scores lead to lower norm scores, which is a violation of the measurement assumptions. 
Obviously, the difficulty of the scale does not allow for differentiation in this performance range of grade five. The example shows that extrapolated norm scores should be used cautiously. Since the derivation shows no other zero-crossing, the regression model seems to be valid within all other ranges of the norm sample.

Please note that the slope depicted here relates to the first partial derivative of the regression function with respect to $l$ not with respect to $a$. Negative slopes with respect to the explanatory variable, in contrast, are not a sign of model inconsistency. For example, in the case of fluid intelligence, empirical data show an increase in performance during childhood and adolescence but a decrease (i.e., negative slope) after the age of 25 years [24]. This declining trajectory over age can usually be modelled with cNORM without problem. Therefore, the norming results should also be validated from a theoretical point of view regarding the measured latent ability.

\subsection{Validation in Terms of Model Fit}

For validation in terms of different information criteria, the 'printSubset(model)' method can be used. The method returns a table containing adjusted $R^{2}$, Mallow's $C_{P}$, $A I C$ and BIC for the best models chosen by the best subset regression in dependence of the number of contained parameters (Figure 8).

\# Getting information criteria for best models with number of predictors \# varying from one to $\mathrm{k}^{2}+2 \mathrm{k}$ numbers of predictors printSubset(model)

\begin{tabular}{r|r|r|r|r|r|r|}
\hline \multicolumn{1}{l|l}{ R2 } & R2adj & \multicolumn{1}{l|}{ RSS } & \multicolumn{1}{l|}{ RMSE } & \multicolumn{1}{l|}{ BIC } & Terms \\
\hline 0.91980991 & 0.91975255 & 5736.13491 & 2.02416383 & $22,283.52$ & -3518.20894 & 1 \\
\hline 0.98008608 & 0.98005757 & 1424.47669 & 1.0087038 & 4486.42639 & -5461.13827 & 2 \\
\hline 0.99085724 & 0.99083759 & 653.997299 & 0.68347709 & 1307.78653 & -6543.73324 & 3 \\
\hline 0.9914362 & 0.99141164 & 612.583591 & 0.66148296 & 1138.82531 & -6628.07395 & 4 \\
\hline 0.99185022 & 0.99182099 & 582.96774 & 0.64529492 & 1018.5672 & -6690.20471 & 5 \\
\hline 0.9922628 & 0.99222948 & 553.455023 & 0.62874877 & 898.734839 & -6755.69238 & 6 \\
\hline 0.99301041 & 0.99297527 & 499.977056 & 0.59760059 & 679.970785 & -6890.71375 & 7 \\
\hline 0.99338161 & 0.99334354 & 473.425064 & 0.58151592 & 572.360683 & -6959.86552 & 8 \\
\hline 0.99376277 & 0.99372238 & 446.159781 & 0.56452242 & 461.806023 & -7035.66439 & 9
\end{tabular}

Figure 8. Information criteria for best models.

As can be seen from Figure 8, the criterion of $R^{2} \geq 0.99$ is only met when at least three predictors are in included in the regression function. These are the predictors returned by the 'bestModel' method, with a raw score RMSE of 0.641. For a graphical visualisation of the relationship between number of predictors and the model fit, the 'plotSubset' method returns an adequate illustration (Figure 9).

As can be seen here, the cut-off is set to the default value of 0.99 . As to be expected, increasing the number of predictors to more than three results is only a slight improvement of the adjusted $R^{2}$. Therefore, the number of three or four terms chosen by the 'bestModel' method seems to deliver a sufficient norming model, while smoothing the percentile curves and cleaning sampling error variance. To investigate possible over- or under-fitting of the model with four predictors, cross validation of the regression model (function 'cnorm.cv') can be used:

\# Cross validation, repeated 10 times with an upper limit of 12 terms cnorm.cv(preparedData, repetitions $=10, \max =12$ )

By using this method, the data set is divided into a training set containing $80 \%$ of the complete sample and a validation sample with $20 \%$ of the norming sample. Subsequently, the best regression models are computed with increasing numbers of terms starting from one until the specified number of predictors (by 'max' parameter). Finally, for every 
resulting training model, the model is projected on the validation data to independently estimate the model fit for data not included in the modelling process. This procedure is repeated $\mathrm{n}$ times (in this example: ten times), and the results are visualised, as can be seen in Figure 10.

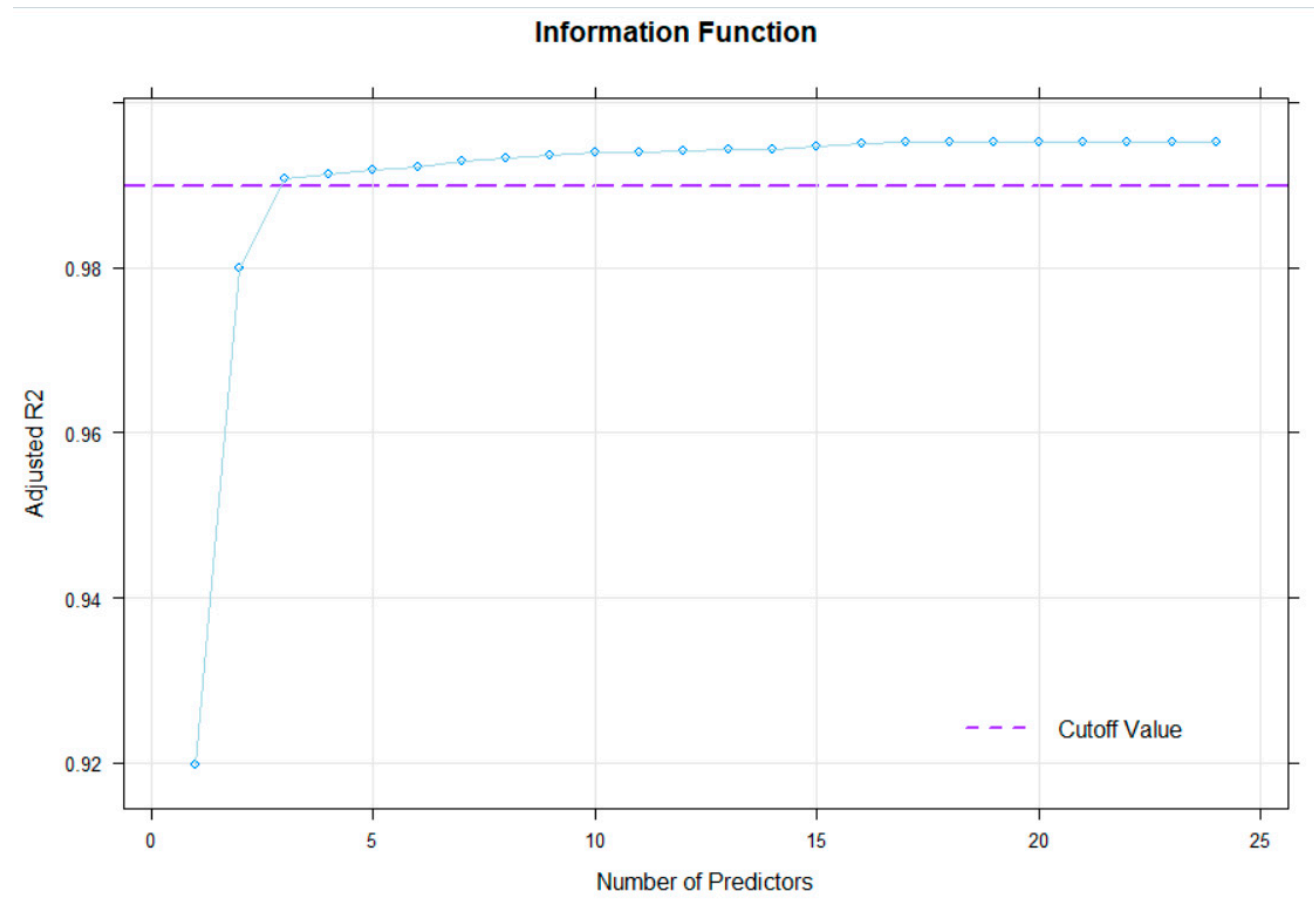

Figure 9. Explained variance depending on number of predictors.

While none of the models shows serious signs of over- or under-fitting, $R^{2}$ of the norm scores increases only slightly when more than four predictors are used in the regression model, i.e., the model can barely be improved by including more than four predictors. This finding seems to be consistent with the aforementioned output of the 'printSubset' method. Therefore, the selected regression model is an adequate, parsimonious and efficient norming model.
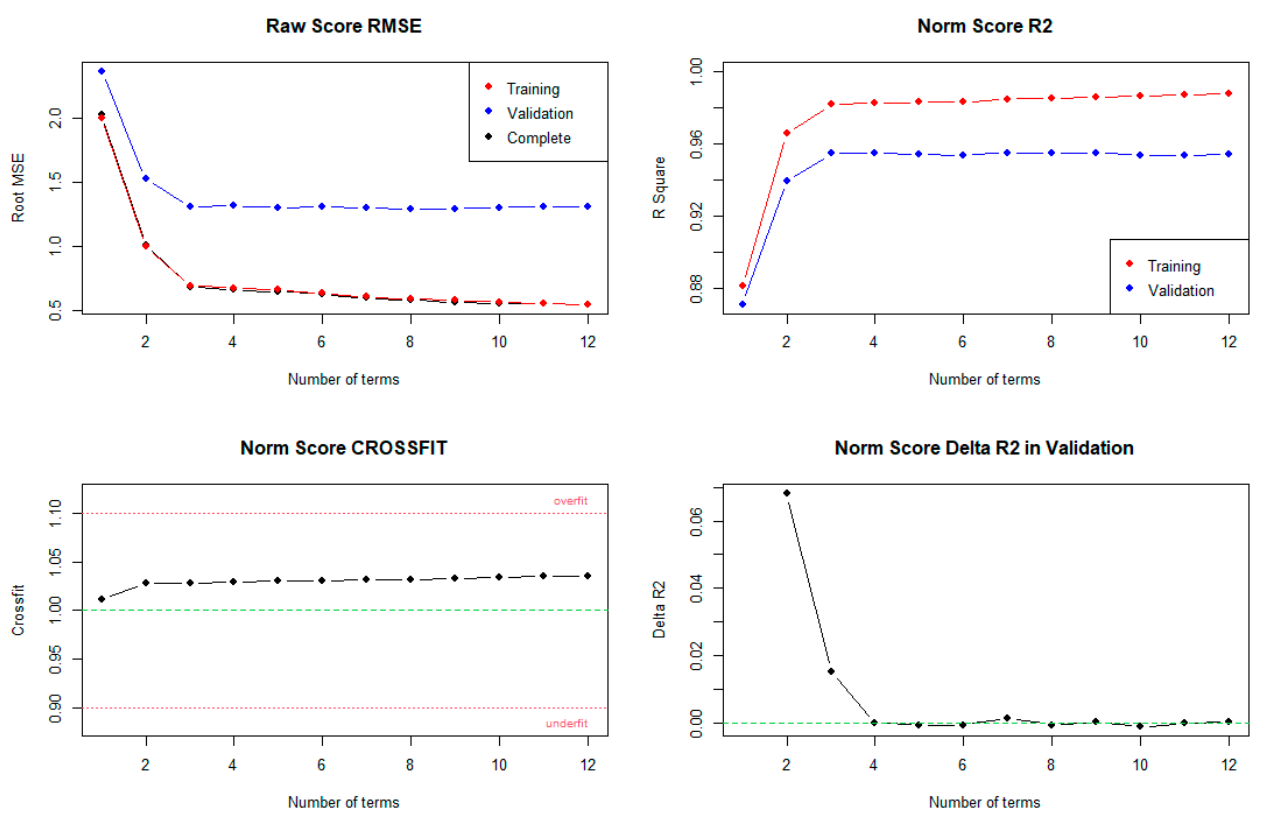

Figure 10. Results of cross validation. 


\subsection{Generating Norm Tables}

After selecting an adequate norming model, the final step of the norming procedure is to generate norm tables, which should be included in the test manual. For this purpose, we choose the 'rawTable' method to transform a series of raw scores, which can be specified by the user, into a series of norm scores as well as corresponding percentiles:

\# Compute raw table for given age between T-scores 25 and 75 for age / grade 3 raw.table <- rawTable $(3$, model, rel $=0.96)$

The table can be restricted in its range by specifying the minimum and maximum norm scores (minNorm, maxNorm) as well as the minimum and maximum raw scores (minRaw, maxRaw). By default, the function retrieves these boundaries based on the values in the data set. Increasing these values leads to extrapolation and elevates the risk of model violations. By specifying the scale reliability and confidence coefficient, the confidence intervals are included in the output both for the norm scores and the percentiles. Therefore, the lower and upper bounds are estimated by

$$
l_{\alpha ; 1-\alpha}=l \pm z_{\alpha ; 1-\alpha} \times \sqrt{r e l \times(1-r e l)} .
$$

The resulting raw table is illustrated in Figure 11.

\begin{tabular}{|r|l|r|r|r|r|r|}
\hline raw & norm & percentile & lowerCI & upperCI & lowerCI_PR & upperCI_PR \\
\hline 0 & 22.91110 & 0.3375439 & 20.77142 & 27.21790 & 0.1734172 & 1.135704 \\
\hline 1 & 24.89777 & 0.6032749 & 22.67862 & 29.12510 & 0.3146237 & 1.842194 \\
\hline 2 & 26.87863 & 1.0385077 & 24.58025 & 31.02673 & 0.5511397 & 2.889239 \\
\hline 3 & 28.85329 & 1.7228978 & 26.47591 & 32.92240 & 0.9326132 & 4.384040 \\
\hline 4 & 30.82133 & 2.7563968 & 28.36524 & 34.81172 & 1.5252292 & 6.440294 \\
\hline 5 & 32.78240 & 4.2556537 & 30.24787 & 36.69435 & 2.4121968 & 9.166608 \\
\hline 6 & 34.73613 & 6.3456755 & 32.12344 & 38.56993 & 3.6915787 & 12.651777 \\
\hline 7 & 36.68216 & 9.1465660 & 33.99164 & 40.43812 & 5.4706581 & 16.948862 \\
\hline 8 & 38.62017 & 12.7563871 & 35.85213 & 42.29861 & 7.8565409 & 22.060868 \\
\hline 9 & 40.54984 & 17.2325394 & 37.70461 & 44.15109 & 10.9434822 & 27.931049 \\
\hline 10 & 42.47086 & 22.5750940 & 39.54879 & 45.99527 & 14.7983490 & 34.440415 \\
\hline 11 & 44.38296 & 28.7158772 & 41.38440 & 47.83088 & 19.4464779 & 41.413850 \\
\hline 12 & 46.28585 & 35.5164217 & 43.21117 & 49.65766 & 24.8606128 & 48.634517 \\
\hline 13 & 48.17929 & 42.7763379 & 45.02887 & 51.47536 & 30.9554814 & 55.864540 \\
\hline 14 & 50.06303 & 50.2514537 & 46.83727 & 53.28375 & 37.5897562 & 62.868597 \\
\hline & & & & & \\
\hline & & & & \\
\hline
\end{tabular}

Figure 11. Raw table for grade level 3.0.

\subsection{In a Nutshell}

Besides these detailed steps, cNORM features convenience methods and standard S3 methods in $\mathrm{R}$ to abridge the process via the general function ' $\mathrm{cnorm}^{\prime}$, as described in the following short syntax:

\# In order to rank the data, compute powers and determine the regression function, the

\# convenience method 'cnorm' can be used with numerical vectors. The resulting object

\# includes the ranked data via object\$data and model via object\$model and it can be used

\# with all plotting methods.

cnorm.elfe <- cnorm(raw = elfe\$raw, group = elfe\$group)

\# Plot R2 of different model solutions in dependence of the number of predictors

plot (cnorm.elfe, "subset", type =0) \# plot R2

plot (cnorm.elfe, "subset", type $=3$ ) \# plot MSE 


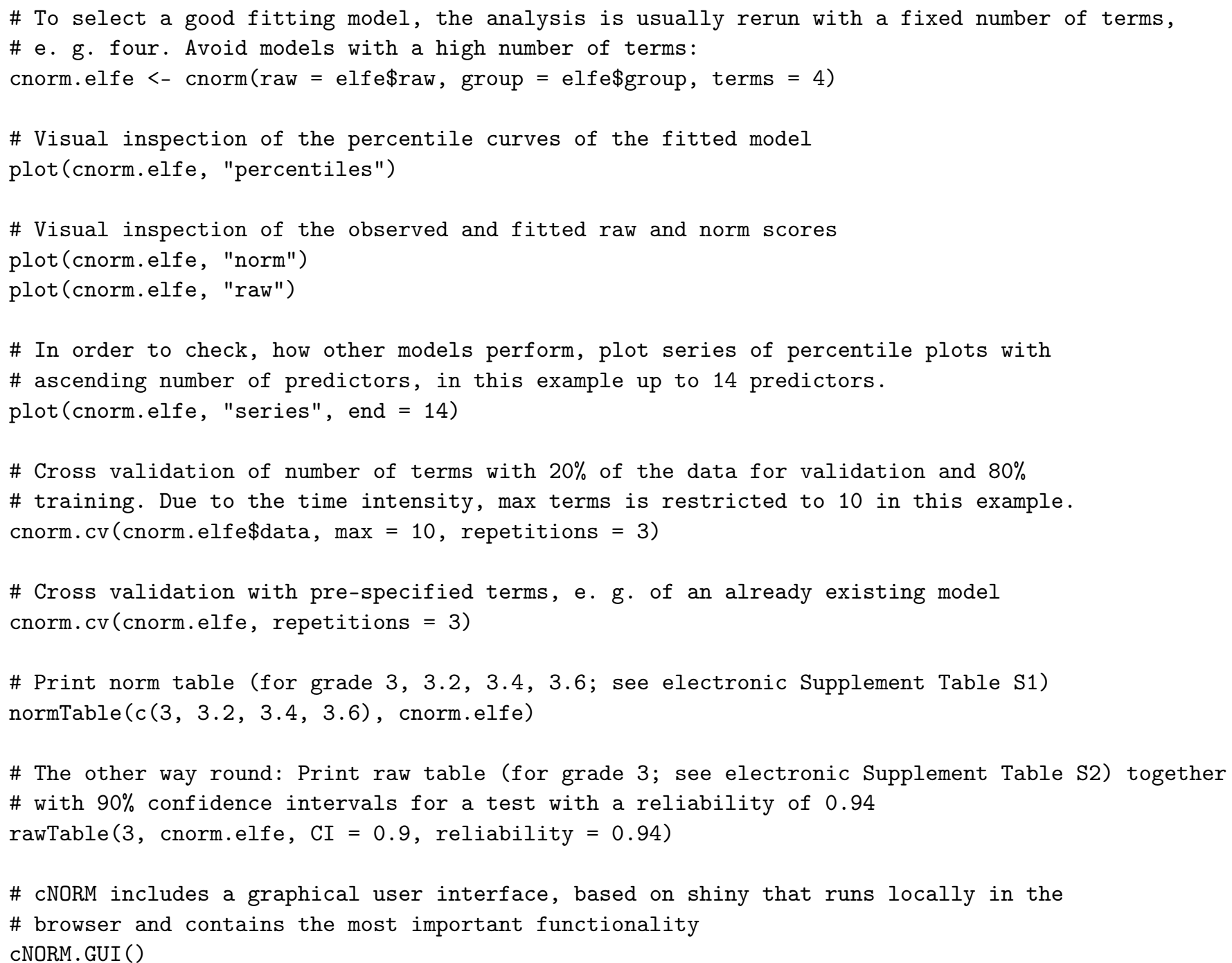

\section{Conclusions}

Norm scores are an essential source for the interpretation of test results when it comes to applied psychometrics and individual diagnostics. The field of norm score generation so far does not capture the full potential of statistical data modelling and largely stays entrenched at the level of a pure description of the manifest data distribution per age group. This shortcoming entails several disadvantages such as high sample size requirements and the inclusion of sampling errors in the resulting norm score tables. Large age intervals in the norm tables can lead to significant bias if the age of a person differs from the average age of the respective subsample. cNORM overcomes or mitigates many of these problems. It allows one to establish regression-based continuous norming models describing the development of a latent variable across explanatory variables such as age. This procedure does not only improve the quality of norm scores but also requires smaller sample sizes of less than 100 cases per norm group, rendering test development more cost efficient [13]. Furthermore, it closes gaps within and between the tables, enables the computation of norm scores for any level of the explanatory variable within the valid range of the norming model and even allows for cautious extrapolation at upper and lower ability levels and beyond the age range of the norm sample. It provides methods for assessing the model fit and the generation of norm tables and enables test users to evaluate the precision of the norm scores. We hope that the package contributes to the design of high quality instruments, improves the precision in individual diagnostics and contributes to progress in the field of norm score modelling. 
Supplementary Materials: The following are available online at https:/ / www.mdpi.com/article/10 .3390 /psych3030033/s1, Table S1: normTable, Table S2: rawTable.

Author Contributions: The original method was developed by A.L. All authors of this manuscript contributed to the development of the cNORM package. With respect to this article, all authors participated in conceptualisation, methodology, software, validation, formal analysis, writing original draft preparation, review and editing and visualisation. All authors have read and agreed to the published version of the manuscript.

Funding: This research received no external funding.

Institutional Review Board Statement: Not applicable.

Informed Consent Statement: Not applicable.

Data Availability Statement: The cNORM package and the example data sets are freely available via CRAN, the according GitHub repository and https:/ / www.psychometrica.de/cNorm_en.html (29 August 2021). The software is licensed under the GNU Affero General Public License v3 (AGPL3.0).

Conflicts of Interest: The authors declare no conflict of interest.

\section{References}

1. Lenhard, A.; Lenhard, W.; Gary, S. Continuous norming of psychometric tests: A simulation study of parametric and semiparametric approaches. PLoS ONE 2019, 14, e0222279. [CrossRef] [PubMed]

2. Timmerman, M.E.; Voncken, L.; Albers, C.J. A tutorial on regression-based norming of psychological tests with GAMLSS. Psychol. Methods 2021, 26, 357-373. [CrossRef] [PubMed]

3. Oosterhuis, H.E.M. Regression-Based Norming for Psychological Tests and Questionnaires. Ph.D. Dissertation, Tilburg University, Tilburg, The Netherlands, 2017.

4. Wei, Y.; Pere, A.; Koenker, R.; He, X. Quantile regression methods for reference growth charts. Statist. Med. 2006, 25, 1369-1382. [CrossRef]

5. Zachary, R.A.; Gorsuch, R.L. Continuous norming: Implications for the WAIS-R. J. Clin. Psychol. 1985, 41, 86-94. [CrossRef]

6. Gary, S.; Lenhard, W. In norming we trust-Verfahren zur statistischen Modellierung kontinuierlicher Testnormen auf dem Prüfstand. Diagnostica 2021. [CrossRef]

7. Koenker, R.; Hallock, K.F. Quantile Regression. J. Econ. Perspect. 2001, 15, 143-156. [CrossRef]

8. Lenhard, A.; Lenhard, W.; Suggate, S.; Segerer, R. A Continuous Solution to the Norming Problem. Assessment 2018, 25, 112-125. [CrossRef] [PubMed]

9. Lenhard, W.; Lenhard, A.; Gary, S. cNorm-Generating Continuous Test Norms. 2018. Available online: https://www. psychometrica.de/cNorm.html (accessed on 4 April 2019).

10. Moosbrugger, H.; Schermelleh-Engel, K.; Gäde, J.C.; Kelava, A. Testtheorien im Überblick. In Testtheorie und Fragebogenkonstruktion, 3rd ed.; Moosbrugger, H., Kelava, A., Eds.; Springer: Berlin, Germany, 2020; pp. 251-273.

11. Goldhammer, F.; Hartig, J. Testwertinterpretation, Testnormen und Testeichung. In Testtheorie und Fragebogenkonstruktion, 3rd ed.; Moosbrugger, H., Kelava, A., Eds.; Springer: Berlin, Germany, 2020; pp. 171-195.

12. Brosius, H.-B.; Haas, A.; Koschel, F. Methoden der Empirischen Kommunikationsforschung: Eine Einführung, 7th ed.; Springer: Berlin/Heidelberg, Germany, 2016.

13. Lenhard, W.; Lenhard, A. Improvement of norm score quality via regression-based continuous norming. Educ. Psychol. Meas. 2021, 81, 229-261. [CrossRef]

14. James, G.; Witten, D.; Hastie, T.; Tibshirani, R. An Introduction to Statistical Learning; Springer: Berlin/Heidelberg, Germany, 2013.

15. Miller, A. Subset Selection in Regression; Chapman and Hall: London, UK, 2002.

16. Zhang, Z. Variable selection with stepwise and best subset approaches. Ann. Transl. Med. 2016, 4, 136. [CrossRef] [PubMed]

17. Hofmann, M.; Gatu, C.; Kontoghiorghes, E.J.; Colubi Cervero, A.M.; Zeileis, A. Lmsubsets: Exact variable-subset selection in linear regression for R. J. Stat. Softw. 2020. [CrossRef]

18. Lumley, T.; Diehr, P.; Emerson, S.; Chen, L. The importance of the normality assumption in large public health data sets. Annu. Rev. Public Health 2002, 23, 151-169. [CrossRef] [PubMed]

19. R Core Team. R: A Language and Environment for Statistical Computing; R Foundation for Statistical Computing: Vienna, Austria, 2020.

20. Lenhard, A.; Lenhard, W. cNORM_Installation. Available online: https://www.psychometrica.de/cNorm_installation_en.html (accessed on 19 June 2021).

21. Moosbrugger, H.; Kelava, A. (Eds.) Testtheorie und Fragebogenkonstruktion: Mit 66 Abbildungen und 41 Tabellen, 2nd ed.; Springer: Berlin/Heidelberg, Germany, 2012. 
22. Döring, N.; Bortz, J. Forschungsmethoden und Evaluation in den Sozial und Humanwissenschaften, 5th ed.; Springer: Berlin/Heidelberg, Germany, 2016.

23. Lenhard, W.; Schneider, W. ELFE 1-6: Ein Leseverständnistest für Erst-Bis Sechstklässler; Hogrefe Göttingen: Göttingen, Germany, 2006.

24. Bugg, J.M.; Zook, N.A.; DeLosh, E.L.; Davalos, D.B.; Davis, H.P. Age differences in fluid intelligence: Contributions of general slowing and frontal decline. Brain Cogn. 2006, 62, 9-16. [CrossRef] 